In vivo Toxicity Assessment of Silver Nanoparticles in Homeostatic versus Regenerating Planarians

Peer-reviewed author version

LEYNEN, Nathalie; VAN BELLEGHEM, Frank; WOUTERS, Annelies; BOVE, Hannelore; PLOEM, Jan-Pieter; THIJSSEN, Elsy; LANGIE, Sabine; CARLEER, Robert; AMELOOT, Marcel; ARTOIS, Tom \& SMEETS, Karen (2019) In vivo Toxicity Assessment of Silver Nanoparticles in Homeostatic versus Regenerating Planarians. In: Nanotoxicology, 13 (4), p. 475-491.

DOI: $10.1080 / 17435390.2018 .1553252$

Handle: http://hdl.handle.net/1942/28319 


\section{In Vivo Characterization and Toxicity Assessment of Silver Nanoparticles in Homeostatic versus Regenerating Planarians}

Silver nanoparticles (AgNPs) currently belong to the most commercialized nanomaterials, used in both consumer products and medical applications. Despite its omnipresence, in-depth knowledge on the potential toxicity of nanosilver is still lacking, especially for developing organisms. Research on vertebrates is limited due to ethical concerns, and planarians are an ideal invertebrate model to study the effects of AgNPs on stem cells and developing tissues in vivo, as regeneration mimics development by triggering massive stem cell proliferation. Our results revealed a strong interference of AgNPs with tissue- and neuroregeneration which was related to an altered stem cell cycle. The presence of a PVP-coating significantly influenced toxicity outcomes, leading to elevated DNAdamage and decreased stem cell proliferation. Non-coated AgNPs had an inhibiting effect on stem cell and early progeny numbers. Overall, regenerating tissues were more sensitive to AgNP toxicity, and careful handling and appropriate decision making is needed in AgNP applications for healing and developing tissues. We emphasize on the importance of AgNP characterization, as we showed that changes in physicochemical properties influence toxicity. 


\section{Introduction}

Silver nanoparticles (AgNPs) are currently the most widely used nanoparticles in consumer products such as food containers (Carbone et al., 2016), clothing (Lee et al., 2007), toothpaste and cosmetics (Benn et al., 2010). Because of their antibacterial and anti-inflammatory properties, the advantages of AgNPs have been intensively explored for diverse medical applications (Chen and Schluesener, 2008, Dakal et al., 2016). They are considered beneficial for tissue regeneration and wound repair (Kumar et al., 2018) and are explored as a treatment for diseases such as leukemia (Guo et al., 2013) and HIV (Elechiguerra et al., 2005).

The high production volumes, use and waste disposal of AgNP-containing consumables inevitably result in the release of AgNPs into the environment (Benn et al., 2010, McGillicuddy et al., 2017, Peters et al., 2018), raising concerns regarding the impact on the environment and human health. Risk assessment, however, is not evident, as variable effects on the cyto- and genotoxic potential of AgNPs are reported (El Yamani et al., 2017). Moreover, developing organisms and tissues are often more susceptible to chemical compounds and particles (Neal-Kluever et al., 2014, Falck et al., 2015). In stages of rapid cell division, stem cells are highly vulnerable to environmental chemicals, which can lead to adverse effects on differentiation processes and development (Perera and Herbstman, 2011). In vitro stem cell toxicity studies report contradictory results on AgNP toxicity. Liu et al. (2015) showed that AgNPs cause oxidative stress and a cell proliferation stop in embryonic neural stem cells in vitro, indicating adverse effects on neurodevelopment. Likewise, Park et al. (2011) linked the generation of reactive oxygen species (ROS) and DNA damage to the inhibition of stem cell proliferation in two different stem cell types. Sengstock et al. (2014) found an impaired osteogenic differentiation of human mesenchymal stem cells (hMSCs) by $80 \mathrm{~nm}$ AgNPs. On the other hand, no change in cell differentiation was observed in human adipose-derived stem cells exposed to 10 and $20 \mathrm{~nm}$ AgNPs (Samberg et al., 2012), or in 
hMSCs exposed to polyvinylpyrrolidone (PVP)-coated $30 \mathrm{~nm}$ AgNPs (He et al., 2016a). An important factor hampering a straightforward comparison of toxicity, is the large variety in physicochemical properties of the NPs under study (McShan et al., 2014). A thorough investigation of properties such as size (Cho et al., 2018), shape (Abramenko et al., 2018) and agglomeration (Bantz et al., 2014) of AgNPs before and during exposure is therefore of utmost importance, in order to avoid conflicting results on seemingly similar NPs (Zhu et al., 2013). Moreover, the surface of AgNPs is often functionalized in order to selectively influence their properties. A protective coating can help in preventing agglomeration of the particles in suspension (de Lima et al., 2012), but the behavior and toxicity of NPs in biological systems might be affected by the addition of a coating (Nguyen et al., 2013). Silver nanoparticles are generally stabilized with PVP, a synthetic polymer that is considered physiologically inactive (Liu et al., 2013).

In this study, we used the freshwater planarian Schmidtea mediterranea to study underlying mechanisms of nanoparticle toxicity on stem cells, regeneration and development in vivo. Planarians have gained interest as toxicological models for compounds such as carcinogens or neurotoxicants (Inden et al., 2004, Gentile et al., 2011, Hagstrom et al., 2015, Stevens et al., 2017). Recently, they were established as a model for assessing nanotoxicity in studies on boron nitride NPs (Salvetti et al., 2015) and AgNPs (Kustov et al., 2014). Because of their large pool of pluripotent stem cells, planarians can regenerate any damaged or amputated tissue, including the entire nervous system, which makes them attractive organisms for (neuro-)developmental research (Hagstrom et al., 2016). In addition, as regeneration is induced by amputation, adults can be studied in parallel with genetically identical regenerating animals. We exposed the worms to non-coated (NC-AgNPs) and PVP-coated (PVP-AgNPs) spherical AgNPs with a nominal size of $20 \mathrm{~nm}$ (actual primary particle size: \pm $35 \mathrm{~nm}$ ) to address changes in physicochemical parameters and toxic effects related to the 
addition of a coating. Homeostatic animals were compared to regenerating animals in order to specifically screen for stem cell-related and developmental effects. To ensure that our results were reproducible and meaningful, an important part of this study was devoted to the determination of the particle characteristics and cellular uptake, in vivo. This enabled us to link variations in physicochemical parameters during AgNP exposure with the observed effects.

\section{Materials and Methods}

\section{Silver Nanoparticle Suspension and Characterization}

Non-coated (NC-AgNPs; Ag = 99.99\%) and polyvinylpyrrolidone-coated (PVP-AgNPs; $\sim 0.2 \mathrm{wt} \%$ ) spherical AgNPs with a nominal size of $20 \mathrm{~nm}$ were purchased from US Research Nanomaterials Inc (Houston, USA). The average diameter and structure of the AgNPs was studied with a transmission electron microscope (TEM; Philips EM 208 S), equipped with a TEM CCD camera (Morada Soft Imaging System). TEM samples were prepared by placing a small drop of AgNPs stock solution onto carbon coated copper grids and allowing it to dry.

Stock solutions of NC- and PVP-AgNPs were prepared by dispersing the NPs in MilliQ water to a concentration of $5 \mathrm{~g} / \mathrm{l}$, followed by sonication for $2 \times 5 \mathrm{~min}$ (probe sonicator, Analis, Belgium, amplitude $18 \mu \mathrm{m}$ ) to avoid agglomeration. In between the cycles, an ice bath reduced suspension heating during sonication. The stock solutions were stored until use (within 1 week) at room temperature and in the dark. Prior to exposure, the AgNP stock solution was sonicated for $5 \mathrm{~min}$ and diluted with culture medium to the preferred exposure concentration, followed by sonication for 3 x $10 \mathrm{~min}$ and cooling for $10 \mathrm{~min}$ on ice in between cycles. The actual exposure concentrations were analyzed with inductively coupled plasma optical emission spectrometry (Optima 8300, PerkinElmer, USA), both undiluted and diluted 1:10 in 2\% nitric acid $\left(\mathrm{HNO}_{3}, 69-70 \%\right.$, J.T. Baker, The Netherlands). 
The intensity-based hydrodynamic diameter $\left(\mathrm{d}_{\mathrm{H}}\right)$, the zeta potential $(\zeta)$ and the dispersity index (Đ) of the exposure medium was measured via dynamic light scattering (DLS) by using a 90Plus Bi-MAS/ZetaPALS (Brookhaven Instruments Corporation, USA).

\section{Model Organisms and Experimental Design}

An asexual strain of the planarian Schmidtea mediterranea was used as previously described (Pirotte et al., 2015). The last 7 days prior to an experiment, the planarians were starved. Experiments were performed in regenerating and homeostatic animals. Regeneration was induced immediately before exposure by transversally cutting homeostatic animals anterior to the pharynx (mouth part) with an ethanol-sterilized razor blade, creating a head and tail part. Animals were exposed in $1 \mathrm{ml}$ exposure medium per worm, control groups in culture medium were always included. During the 3 and 7 days exposure experiments, the exposure medium was refreshed once and twice respectively. The exposure concentrations (between 5 and 50 $\mathrm{mg} / \mathrm{l})$ were selected based on literature.

In order to compare the toxicity of nanosilver to ionic silver $\left(\mathrm{Ag}^{+}\right)$, the organisms were exposed to equivalent concentrations of 5 - $50 \mathrm{mg} / \mathrm{l}$ silver nitrate $\left(\mathrm{AgNO}_{3}\right.$, Sigma Aldrich), using 2.5 - $50 \mathrm{mg} / \mathrm{l} \mathrm{Ca}\left(\mathrm{NO}_{3}\right)_{2}$ (Holeywell Fluka) as a control for the administered nitrate.

To assess a possible disturbance of the ROS balance by AgNPs in exposed animals, we added a water soluble SOD inhibitor to the exposure medium. Diethyldithiocarbamic acid sodium salt (DETC, $0.25 \mu \mathrm{M}$; Sigma Aldrich) was dissolved in culture medium with $5 \mathrm{mg} / \mathrm{l}$ PVP-AgNPs and compared with control animals (medium and medium + DETC) and exposed animals (5 mg/l PVP-AgNPs). Regenerating animals were compared with homeostatic animals, ten animals were used per condition. After 7 days, effects were studied by measuring differences in the unpigmented regeneration bud at the wound side, which is called the blastema. Blastema areas were quantified using ImageJ (version 1.48v) on digital micrographs of gliding animals acquired with a Nikon Cs-Ri2 digital camera mounted on a Nikon SMZ800 
stereomicroscope. Each blastema area is an average of three independent measurements, normalized against the total body area of the worms.

\section{Silver Nanoparticle Uptake and Distribution}

Worms were exposed to 5 and 15 mg/l NC- and PVP-AgNPs for 7 days (homeostatic worms) and for 1 and 3 days (regenerating worms). The silver concentration in the different water phases and worms was determined by inductively coupled plasma mass spectrometer (ICPMS) after 1, 3 or 7 days of exposure. Five individual worms were transferred to a $1.5 \mathrm{ml}$ tube and samples were processed as described by Gray et al. (2013), with adjustments. A full description can be found in Supplement 1. The particle number concentration, particle size distribution and ionic concentration were measured using a NexION 350S ICP-MS (PerkinElmer, USA) in single particle mode (SP-ICP-MS). Silver concentrations were determined for each phase (epidermal mucus, wash water, worms, medium and sediment).

To characterize the distribution pattern of AgNPs in the worms, homeostatic animals were exposed to PVP-AgNPs (15 mg/l, 10 days), followed by mucus removal with 2\% $\mathrm{HCl} / \mathrm{PBS}$. Next, the animals were fixed and mounted in glycerol. AgNPs were localized by their plasmonic-excited fluorescence in whole mount using confocal laser-scanning microscopy (CLSM), combined with a nuclei- and cell membrane specific counterstain. Regenerating animals were exposed to 5 mg/l PVP-AgNPs for 3 days. Animals were killed in 5\% N-acetyl-L-cysteine (NAC) in phosphate buffered saline (PBS) for $7 \mathrm{~min}$, which also removes the mucus layer. The worms were fixed with $4 \%$ deionized formaldehyde (FA) in PBS for 25 min while shaking, the solution was refreshed once after $10 \mathrm{~min}$. After two washing steps with PBS (4 x $10 \mathrm{~min}$ ) and 50\% MeOH in PBS (10 min), the samples were incubated in $100 \% \mathrm{MeOH}$ at $-20^{\circ} \mathrm{C}$ for at least $1 \mathrm{~h}$, and washed with $50 \% \mathrm{MeOH}$ in PBS (2 x $10 \mathrm{~min})$ and PBS (10 min). Membrane and nuclei counterstaining was performed overnight with $4 \mu \mathrm{g} / \mathrm{ml}$ Wheat Germ Agglutinin Alexa Fluor ${ }^{\mathrm{TM}} 488$ Conjugate (WGA, Invitrogen, 
cellular membranes) and $5 \mu \mathrm{M}$ Vybrant DyeCycle Ruby stain (Invitrogen, nuclei) in PBS. The stained samples were rinsed 6 x 10 min with PBS and mounted in Shando Immu-Mount (Thermo Fisher Scientific, Germany). CLSM was performed using a Zeiss LSM510 META confocal microscope mounted on an Axiovert 200M (Zeiss, Germany). Specific information on laser types and band-pass filters can be found in Supplement 2. Image acquisition was performed with the ZEN software version 2.3 (Zeiss) and processed with the image processing package Fiji (ImageJ v1.52e, Open source software, http://fiji.sc/Fiji).

\section{Morphological Characterization of Tissue Regeneration}

Nanosilver-related effects on regenerative capacity were studied by measuring differences in the blastema. The blastema area of twenty regenerating head and tail parts, unexposed and exposed to $15 \mathrm{mg} / \mathrm{l} \mathrm{NC-AgNPs}$ and PVP-AgNPs, were measured daily from 4 - 7 days post amputation (dpa). Blastema areas were measured and processed in the same way as described earlier.

To study the regeneration of photoreceptors, twenty worms were decapitated and exposed to $15 \mathrm{mg} / \mathrm{l} \mathrm{NC-AgNPs} \mathrm{and} \mathrm{PVP-AgNPs.} \mathrm{According} \mathrm{to} \mathrm{our} \mathrm{experience,} \mathrm{planarian} \mathrm{eye}$ regeneration takes place from the $5^{\text {th }}$ dpa. Worms were scored on the presence of photoreceptors and on their clarity (faint or clear) on the $7^{\text {th }}$ dpa.

\section{Immunohistochemistry}

In order to study effects of AgNP exposure on the stem cell proliferation, the in vivo mitotic activity was examined using an immunohistochemical staining with anti-phospho-Histone H3 (H3P) antibody, as described previously (Pirotte et al., 2015). The analysis of mitosis was performed on at least six homeostatic and regenerating animals per experiment, which were exposed to $50 \mathrm{mg} / \mathrm{l}$ for 7 days and 5 - $35 \mathrm{mg} / \mathrm{l}$ for 1 and 3 dpa, respectively. Samples were digitized and the mitotic stem cell number was counted with the Nikon imaging software 
(NIS-Br). The number of mitotic stem cells was normalized against the total body area of the worms.

Neurodevelopment was studied by decapitating at least eight animals per experiment and studying the regenerating head at 7, 10 or 14 dpa. Exposure concentrations ranged from 5 - 15 mg/l. To study cephalic brain ganglia degeneration, at least seven homeostatic animals per condition were exposed for 7 or 14 days to 15 and $5 \mathrm{mg} / \mathrm{l}$, respectively. Samples were stained with an anti-synapsin 3C11 antibody (Developmental Studies Hybridoma Bank, developed by Buchner E.) as described by Pirotte et al. (2015). The relative brain widths were measured as described by Hagstrom et al. (2015) (schematic representation in figure 2D). Measurements were performed with ImageJ (version 1.48v) on digital images acquired with a Nikon Cs-Ri2 digital camera mounted on a Nikon SMZ800 stereomicroscope.

\section{Fluorescent in situ Hybridization}

The fluorescent in situ hybridization protocol is based on King and Newmark (2013), and modified as described by Stevens et al. (2017). Additional adjustments in the protocol or experimental specifications are mentioned here. At least five regenerating heads and tails per experiment were exposed for 3 days to 5 mg/l NC- and PVP-AgNPs. Animals were killed and fixed, after which they were washed in PBST (PBS + 0.3\% Triton X-100) and rinsed in 50\% $\mathrm{MeOH} / \mathrm{PBST}$ and $100 \% \mathrm{MeOH}$. They were incubated at least $1 \mathrm{~h}$ at $-20^{\circ} \mathrm{C}$ in $100 \% \mathrm{MeOH}$. Samples were rehydrated and incubated in 1x saline-sodium citrate (SSC, in MilliQ water). Pigmentation was removed after $2 \mathrm{~h}$ in deionized formamide bleaching solution (5\% formamide, $0.5 \mathrm{x}$ SSC and $1.2 \% \mathrm{H}_{2} \mathrm{O}_{2}$ ). $1 \mathrm{xSSC}$ was added for 5 min, followed by PBST washes. Proteinase K solution for permeabilization was added for 7-8 min. Animals were again fixed and washed in PBST. A 50\% prehybridization/PBST solution (without heparin nor DTT) was added for $10 \mathrm{~min}$, followed by fresh prehybridization solution for $2 \mathrm{~h}$, at $56^{\circ} \mathrm{C}$. Samples were incubated in 1-5 ng probe per $\mu$ l hybridization solution (prehybridization 
solution $+5 \%$ dextran sulphate) for about $18 \mathrm{~h}$ at $56^{\circ} \mathrm{C}$. Probes were synthesized according to Stevens et al. (2017), being Smedwi-1 (Reddien et al., 2005), a marker for dividing adult stem cells, and SmedNB21.11e, a marker for early stem cell progeny (Eisenhoffer et al., 2008). Probes were removed by a series of washing steps with decreasing concentrations of SSC at $56^{\circ} \mathrm{C}$. Samples returned to room temperature in 2 x 10 min TNTx buffer solution ( $0.1 \mathrm{M}$ Tris, $0.15 \mathrm{M} \mathrm{NaCl}$ and $0.3 \%$ Triton $\mathrm{X}-100, \mathrm{pH}=7.5)$. Non-specific binding sites were blocked with blocking solution (5\% horse serum and 0.5\% Roche Western Blocking Reagent diluted in TNTx) for 1.5 - 2 h. An anti-Digoxigenin-POD antibody (Roche, USA) in blocking solution (1:500) was added overnight $\left(4^{\circ} \mathrm{C}\right)$. Samples were washed repeatedly in TNTx. Tyramide signal amplification (TSA) mix was made by dissolving FAM fluorescein (1:500), 4iodophenylboronic acid (1:100) and $\mathrm{H}_{2} \mathrm{O}_{2}(0.003 \%)$ in TSA buffer (2M NaCl and $0.1 \mathrm{M}$ boric acid; $\mathrm{pH}=8.5$; sterilized). TSA mix was added to the samples for $15 \mathrm{~min}$, followed by washing steps in TNTx and PBST. A counterstain with $5 \mu \mathrm{M}$ Vybrant DyeCycle Ruby stain (Invitrogen) in PBST was added overnight $\left(4^{\circ} \mathrm{C}\right)$, followed by washing steps with PBST and a postfixation. After some final washing steps with PBST, samples were mounted in Shando Immu-Mount (Thermo Fisher Scientific).

The expression patterns of Smedwi-1 and SmedNB21.11e were visualized using a fluorescent microscope (Nikon Eclipse 80i) with a Nikon Cs-Ri2 digital camera. The number of cells expressing SmedNB21.11e was determined in the heads and in the postpharyngeal area and at the right side of the pharynx in tails with the Nikon imaging software (NIS-Br) and normalized against the total body area of the worms.

\section{COMET Assay}

DNA damage was assessed with the alkaline comet assay, according to Stevens et al. (2017). Additional adjustments in the protocol or experimental specifications are mentioned here. Regenerating animals were exposed to $5 \mathrm{mg} / \mathrm{l} \mathrm{NC}$ - and PVP-AgNPs for 3 days, controls were 
regenerated in cultivation medium. In short, cells were obtained after cell dissociation of one animal per sample (six samples per condition). Samples were filtered through a $35-\mu \mathrm{M}$ filter (Pluriselect). Two comet slides were made of each sample and considered to be technical repetitions. Electrophoresis was carried out at $1 \mathrm{~V} / \mathrm{cm}(\sim 0.95 \mathrm{~V} / \mathrm{cm})$ over the platform, for 20 min at $4^{\circ} \mathrm{C}$. Image analysis was performed with a Zeiss fluorescence microscope (AxioImager.Z2, equipped with SlideFeeder X80) using the 10x objective magnification. Comet images were automatically captured with Metafer 5 (Metasystems) and subsequently analyzed with the Comet assay IV (Instem - Perceptive instruments) to assess the amount of DNA damage. The tail intensity, being the \% DNA in the tail relatively to the total amount of DNA in the comet, as well as tail moment (the product of the \% tail DNA and the tail length) were calculated by the software and used for further data analysis. Median values were calculated per sample and average values were determined of the technical repetitions.

\section{Statistical Analysis}

Groups were statistically compared using a one-way analysis of variance (ANOVA) or a twoway ANOVA followed by a Tukey HSD post-hoc test for multiple comparisons. Normality was tested according to the normality test of Shapiro-Wilk. If the assumptions of normality were not met, a transformation of the data set was applied (Log, Square root, 1/x and $\mathrm{e}^{\mathrm{x}}$ ). All statistical analyzes were performed using RStudio 0.98 .1103 (Rstudio, Inc.). P-values $<0.05$ were considered significant.

\section{Results}

\section{Characterization of AgNP in Exposure Medium}

Silver nanoparticle characterization was based on shape, primary particle size, $\mathrm{d}_{\mathrm{H}}, \zeta$ and $Đ$ (Table 1). Both NC- and PVP-AgNPs were spherical in shape and approximately 1.5 times the nominal size indicated by the manufacturer (Figure S1(A-B)). Volume-based D10, D50 and D90 sizes correspond to the $10 \%, 50 \%$, and $90 \%$ of particles under the reported $\mathrm{d}_{\mathrm{H}}$. Zeta 
potentials were slightly negative for NC-AgNPs (-9.48 mV) and PVP-AgNPs (-6.79 mV) and the samples were moderately polydisperse. Size distribution for both NC- and PVP-AgNPs in the exposure medium was similar (Figure S2(A-B)) with $37 \mathrm{~nm}$ having the highest frequency (Table 1). Mean sizes were $110 \mathrm{~nm}$ and $101 \mathrm{~nm}$ for NC- and PVP-AgNPs, respectively. After $48 \mathrm{~h}$ (the moment of refreshing), four times more sedimentation was observed for NC-AgNP particles compared to PVP-AgNPs. In an additional experiment, AgNP medium without worms was analyzed (Table 2). The concentrations of NC-AgNPs and PVP-AgNPs in the water column were three to seven times higher compared with the medium containing worms. No $\mathrm{Ag}^{+}$was detected in both media after $48 \mathrm{~h}$.

\section{Particle Uptake and Distribution in the Organism}

The particle size distribution and concentration in regenerating worms were measured at 1 and 3 dpa. All measurements, unless indicated otherwise, are the results of at least two independent experiments. The size distributions of silver in the worms was smaller compared to that of the exposure medium (Figure S2(C-D). The most frequent particle size in the worms exposed to $15 \mathrm{mg} / \mathrm{l}$ during 3 days was similar in worms exposed to NC- and PVP-AgNPs, with a decrease in mean particle size between 1 day and 3 days of exposure (Figure 1(A)). Silver was detected in the epidermal mucus in concentrations up to $0.083 \mu \mathrm{g} / \mathrm{l}$ (Figure 1(A)). Silver concentrations significantly increased in function of exposure concentration and time (p $<0.001$ ) (Figure 1(B)). Homeostatic worms contained similar concentrations of both NP types after 7 days of exposure (Figure 1(B)), with mean sizes ranging from 29 to $48 \mathrm{~nm}$ (NCAgNPs) and from 28 to 49 nm (PVP-AgNPs). Both NP types exhibit an absorption maximum of $405 \mathrm{~nm}$ and a broad emission band from 400 to $700 \mathrm{~nm}$, which was used to identify their internal tissue and cellular distribution by confocal microscopy (Figure S3(A)). No specific distribution pattern could be determined within the body, although a fraction of the NPs seems to accumulate in the gut (Figure S3(B)). The intracellular uptake of both NPs was evident 
from their presence inside the cytoplasm (Figure 1(C)). Particles were observed in the immediate area around the nucleus (Figure S3(C)). By means of fluorescent stem cell staining, we were able to locate both NP types in or around stem cells (Smedwi-1, Figure 1(D)) and early progeny (SmedNB21.11e, Figure 1(E)).

\section{Physiological and Morphological Alterations in Regenerating Animals due to AgNP}

\section{Exposure}

Tissue regeneration (blastema formation, photoreceptor appearance and the formation of the cephalic ganglia) was monitored over time. A timeline of the experiments is represented in Figure 2(A).

Tissue Regeneration is Disrupted by PVP-coated AgNPs

The anterior and posterior blastema sizes of PVP-AgNP-exposed worms were significantly smaller (15 mg/l, p < 0.001) compared to non-exposed worms (Figure 2(B)). The inhibiting effect on anterior regeneration was confirmed at a lower PVP-AgNP concentration (5 mg/l, p $=0.001$, Figure $4(\mathrm{~A}))$. In 59\% of the PVP-AgNP-exposed animals, eyespots were partially regenerated (33\%) or absent (26\%) (Figure 2(C)). In case of NC-AgNP exposure, photoreceptor regeneration was disrupted in $24 \%$ of the animals.

PVP-coated AgNPs Induce Neurodevelopmental Toxicity

Neurodevelopmental toxicity was assessed via measurements of relative brain sizes after regeneration under NC- and PVP-AgNP exposure for 7 dpa (5 and 15 mg/l), 10 dpa (15 mg/l) and 14 dpa (5 and $15 \mathrm{mg} / \mathrm{l}$ ), as described by Hagstrom et al. (2015) (Figure 2(D)). When considering all experiments, $4.35 \%$ of regenerating animals exposed to NC-AgNPs and $9.30 \%$ of those exposed to PVP-AgNPs failed to regenerate their cephalic brain ganglia. At 7 dpa, the lowest NC-AgNP exposure resulted in a significantly larger relative brain size, compared to the non-exposed worms $(\mathrm{p}=0.036)$. When exposed to the highest exposure concentration, $10 \%$ of NC-AgNP- and 29\% of PVP-AgNP-exposed animals couldn't regenerate their 
cephalic brain ganglia. In the other animals, PVP-AgNP exposure resulted in a decreased relative brain width $(\mathrm{p}=0.022)$. At $10 \mathrm{dpa}$, 8\% of NC-AgNP- and 17\% of PVP-AgNPexposed animals did not regenerate a new brain, however no changes in brain size were observed in the organisms who did. At $14 \mathrm{dpa}$, all animals regenerated a brain and only the highest concentration interfered with brain regeneration, resulting in smaller brains in both NC-AgNP- $(\mathrm{p}<0.001)$ and PVP-AgNP-exposed $(\mathrm{p}<0.001)$ worms.

Exposure to both types of AgNPs decreased the motility of regenerating organisms and changed the type of motility behavior in a concentration-dependent way. The most prominently displayed change in behavior was a shift from gliding (Video S4(A)) to dragging (Video S4(B)). Other observed behavioral alterations were curling, screw-like movements, side lying and immobility (Figure S5(A-B)). Behavioral effects were more pronounced during PVP-AgNP exposure, as compared to the NC-AgNP-exposed animals. On the $3^{\text {rd }} \mathrm{dpa}, 5 \mathrm{mg} / \mathrm{l}$ NC-AgNPs affected about $7 \%$ of the exposed animals, whereas $20 \%$ was affected when exposed to $5 \mathrm{mg} / \mathrm{l}$ PVP-AgNPs.

\section{Cellular and Molecular Alterations in Regenerating Animals due to AgNP Exposure}

\section{Changes in Stem Cell Dynamics}

No changes in stem cell proliferation were detected in the regenerating heads after one day of exposure. Three days of exposure to $5 \mathrm{mg} / \mathrm{l} \mathrm{PVP-AgNPs} \mathrm{induced} \mathrm{a} \mathrm{significant} \mathrm{decrease} \mathrm{in}$ H3P-positive cells ( $\mathrm{p}=0.005$ ), whereas exposure to NC-AgNPs resulted in a slight, but not significant, proliferation decrease $(p=0.067)$ (Figure $3(A)$ ). Three days of exposure to 15 mg/l NPs resulted in a significantly lower amount of proliferating stem cells for both NCAgNPs ( $=0.002)$ and PVP-AgNPs $(p=0.003)$. Also a 3-day exposure to $35 \mathrm{mg} / \mathrm{l} \mathrm{NC}$ AgNPs, resulted in a significantly declined stem cell proliferation $(\mathrm{p}=0.004)$. In the tail parts, stem cell proliferation was less clearly affected, with only a significant increase after 1 day 
exposure to $15 \mathrm{mg} / \mathrm{l}$ PVP-AgNPs ( $\mathrm{p}=0.018$ ). No effects were seen after 3 days of exposure (Figure S6(A)).

As most differences in stem cell proliferation were found $3 \mathrm{dpa}$, the total amount of stem cells was visualized by Smedwi-1 expression at that time point. Compared to the nonexposed worms, Smedwi-1 expression decreased in the heads of AgNP-exposed worms, with the largest decline in Smedwi-1-expressing cells in NC-AgNP-exposed animals (Figure 3(B)). No differences were detected in the regenerating tails (Figure S6(B)).

Early progeny cells were quantified via SmedNB21.11e-expression (Figure 3(C)). At 3 dpa, NC-AgNP-exposed head parts showed a significant decrease in NB21.11e-expressing cells ( $\mathrm{p}<0.001)$, while PVP-AgNP exposure induced no differences $(\mathrm{p}=0.188)$. SmedNB21.11e-expression in tails was significantly decreased in both AgNP-types ( $\mathrm{p}<$ 0.001) (Figure S6(C)).

\section{Redox-related Alterations}

We functionally evaluated the role of the internal redox status using a SOD inhibitor. Interfering with the redox balance induced significant regeneration defects in head and tail parts ( $\mathrm{p}=0.032$ and $\mathrm{p}<0.001$ respectively). In tail parts, the disturbed regeneration by PVPAgNPs was improved with the simultaneous exposure to the SOD-inhibitor, as a significantly larger blastema was observed compared to the non-exposed SOD-inhibited worms $(\mathrm{p}<0.001)$ (Figure 4(A)). No alterations were seen when PVP-AgNP exposed head parts were simultaneously exposed to a SOD-inhibitor $(\mathrm{p}=0.504)($ Figure S7(A)).

\section{AgNPs Induce DNA Damage}

The amount of DNA damage was significantly elevated in regenerating heads exposed to 5 mg/l PVP-AgNPs during 3 days $(\mathrm{p}=0.028)$, with an average of $12.60 \%$ tail DNA compared to $7.19 \%$ tail DNA in controls (Figure 4(B)). The amount of DNA damage found after NCexposure in heads was also increased (11.49\% tail DNA), though not significant. A similar 
result was seen when calculating the tail moment, which combines tail DNA with the smallest detectable sizes of migrating DNA. A higher number was measured in PVP-exposed heads (2.18) compared to control heads $(0.69, \mathrm{p}=0.045)$, although an elevation was also seen in NC-exposed heads (1.29) (Figure S7(B)). In tails, NC- and PVP-AgNP exposure did not result in a difference in tail moment, compared to controls $(\mathrm{p}=0.739$ and $\mathrm{p}=0.775$, respectively). Higher numbers in tail moment were found in exposed heads, compared to exposed tails $(\mathrm{p}=0.024)$.

\section{High Toxicity of Silver Ions}

To verify if the observed effects could relate to the release of $\mathrm{Ag}^{+}$from AgNPs, regenerating worms were exposed to 5 - $50 \mathrm{mg} / \mathrm{l} \mathrm{AgNO}_{3}$. After a few hours of exposure with $\mathrm{AgNO}_{3}$, the regenerating worms started displaying altered behavior (immobility, dragging), followed by a $100 \%$ mortality within one day. No effects were observed in the control group exposed to $\mathrm{Ca}\left(\mathrm{NO}_{3}\right)_{2}$ (data not shown).

\section{AgNPs Have Little Effects in Homeostatic Animals}

Fully regenerated animals represent a condition of physiological homeostasis, and a comparison with regenerating animals offers the possibility to uncover regenerationassociated effects of AgNPs.

In homeostatic animals, no behavioral changes were observed at the lower exposure concentration (5 mg/l) during 14 days of exposure. Exposure to $15 \mathrm{mg} / \mathrm{l}$ did induce behavioral changes from the $3^{\text {rd }}$ day for PVP-AgNPs (dragging, 30\% of animals), and from the $5^{\text {th }}$ day when exposed to NC-AgNPs (50\% of animals). Screw-like movements and side-lying behavior was only seen at the highest concentrations (50 mg/l).

In contrast to the regenerating animals, AgNPs induced no significant effects on the stem cell proliferation (at $50 \mathrm{mg} / \mathrm{l}$ ), as illustrated in Figure 5(A). No impact of interfering with the redox balance was observed on animal behavior or phenotype when inhibiting SOD 
during NP exposure (data not shown). No brain degradation was noticed in animals exposed to 5 or $15 \mathrm{mg} / \mathrm{l} \mathrm{NC-} \mathrm{and} \mathrm{PVP-AgNPs} \mathrm{during} 7$ or 14 days (Figure 5(B)).

Also in homeostatic animals, exposure to $\mathrm{Ag}^{+}$ions immediately led to altered behavior, with a high incidence of immobility. One day of exposure, even to the lowest $\mathrm{AgNO}_{3}$ concentration, resulted in $100 \%$ mortality.

\section{Discussion}

Nanoparticles are economically attractive because of their small size and high reactivity. After their release into the environment or when taken up by organisms, these particles can undergo physicochemical transformations, resulting in a broad spectrum of particle sizes, particle complexes and properties (Almeida et al., 2011). Because of this variability, regulation on nanoparticle-containing consumables is not straightforward. Especially for children and developing organisms, which are known to be more susceptible, toxicity is not well formulated (Neal-Kluever et al., 2014, Falck et al., 2015). On the other hand, AgNPs are considered as beneficial in tissue regeneration and wound repair (Kumar et al., 2018). These discrepancies emphasize the need to understand possible effects of AgNPs on developing cells and tissues, while taking the physicochemical variations into account.

We used the freshwater flatworm Schmidtea mediterranea to address AgNP-induced effects on tissue development and regeneration. We show that an interdisciplinary approach to study NP toxicity is needed to explain heterogeneous responses. Too little in vivo studies take into account the physicochemical characteristics and the actual exposure concentrations, in order to link them to uptake, distribution patterns and adverse outcomes. For example, the actual size of the AgNPs used in this study was considerably larger than their nominal reported particle size (Figure S1(A-B) and they reached a hydrodynamic diameter op to 268 $\mathrm{nm}$ in suspension (Table 1). Secondly, AgNP-type-dependent changes in both concentration and particle size were observed during the exposure period (Figure 1(A), Table 2). PVP 
coating prevents association to larger aggregates, resulting in smaller particles and a higher concentration in the medium (Table 2, Figure S2(A-B)). Sedimentation also depends on the surrounding environment (Fabrega et al., 2011), such as the presence of chloride in the exposure medium (Li et al., 2010) and the production of organic material such as mucus, in which particles agglomerate (Table 2). This way, the bioavailability in the water column is reduced, while increasing the risk for benthic organisms.

Planarians inhabit the lowest water layer, and can uptake NPs as they glide over the substrate. The observed intestinal accumulation (Figure S3(B)) might be related to a slow depuration rate of the ingested AgNPs (Zhao and Wang, 2011) or to a low distribution to extra-intestinal tissues (Zhao and Wang, 2010, Bergin and Witzmann, 2013). High AgNP concentrations in the digestive tracts are not necessarily correlated to a higher toxicity. Zhao and Wang (2011) demonstrated that Daphnia magna can accumulate extraordinary high intestinal AgNP levels, without obvious signs of toxicity. This was confirmed by Pakrashi et al. (2017), who correlated mortality rates in Daphnia magna with AgNPs accumulation in non-gut tissues. Most likely, the intestinal mucus forms a protective barrier against AgNPs, comparable with the epidermal mucus layer of the worms which appeared to trap and agglomerate AgNPs (Figure 1(A), Table 2). Also in other organisms, mucus has a similar protective function, e.g. in the gills of fish or in the human respiratory system (Courrier et al., 2002, Scown et al., 2010).

In addition, intestinal $\mathrm{pH}$ levels interfere with the particle uptake. Dissolution of AgNPs increases as pH decreases (Behra et al., 2013). Our model organism is expected to have a slightly acidic intestinal pH (<5.5) (Goupil et al., 2016), suggesting a potential release of $\mathrm{Ag}^{+}$in the gut. This can be linked to the observed reduction in mean AgNP sizes inside the organisms, occurring between 1 and 3 days of exposure (Figure 1(A)). Dissolution of $20 \mathrm{~nm}$ AgNPs with PVP surfactant was also observed in the gut of organisms with a more neutral 
pH, such as isopods (Romih et al., 2016). The released silver ions, which can enter cells via ion transporters, are considered to be more toxic than AgNPs (Zhao and Wang, 2011, Bilberg et al., 2012, Mehennaoui et al., 2016, Pakrashi et al., 2017). We did not detect $\mathrm{Ag}^{+}$ions in the exposure medium, suggesting a low exposure to external $\mathrm{Ag}^{+}$. When we externally administered $\mathrm{Ag}^{+}$ions, both regenerating and homeostatic animals reacted very strong, resulting in a $100 \%$ mortality within the first $24 \mathrm{~h}$ at the lowest $\mathrm{AgNO}_{3}$ concentration.

Cellular uptake of Ag particles was confirmed by confocal microscopy (Figure 1(C)) and ICP-analysis revealed a higher bioavailability of the smaller-sized fraction (Figure S2). Particulate silver might be also formed biogenically from $\mathrm{Ag}^{+}$in vivo, as demonstrated in earthworms and rats (van der Zande et al., 2012, Baccaro et al., 2018). Nanosilver and released ions interfere with the regeneration process, resulting in smaller blastema sizes and a delayed regeneration of the photoreceptors. Solely PVP-coated AgNPs strongly disrupted anterior and posterior regeneration and although they are considered to be physiologically inactive (Liu et al., 2013) (Figure 2(B-C)). We further investigated this effect, as PVP-coated AgNPs in combination with chitosan are considered promising in wound healing (Archana et al., 2015). Underlying the disturbed regeneration process lies a significant decrease in stem cell proliferation in the regenerating heads at $3 \mathrm{dpa}$, a point in time considered to be the mitotic maximum (Figure 3(A)) (Baguñà, 1976). The mitotic decrease can at least partially be related to DNA damage, which was mainly detected in heads exposed to PVP-AgNPs (Figure 4(B)). Ahamed et al. (2008) also found higher DNA damage by coated AgNPs in vitro, compared to uncoated AgNPs, which they explained by the more individually distributed particles. Eyeless phenotypes or defective eyes were also observed by Asharani et al. (2011) in developing zebrafish embryos after exposure to polyvinyl alcohol-coated AgNPs (5-20 $\mathrm{nm}$ ), induced after damage to DNA or proteins responsible for eye development. NPs were located near the nuclei (Figure S3(C)), where they can act as a source of $\mathrm{Ag}^{+}$(McShan et al., 
2014), or indirectly induce DNA damage via oxidative stress. In addition to the decreased stem cell proliferation, we observed a decrease in the total amount of stem cells and early progeny cells in the head parts, although this difference was stronger in NC-AgNPs-exposed worms (Figure 3(B-C)). We were able to locate AgNPs in or nearby Smedwi-1 expressing stem cells and early progeny cells (Figure 1(D-E)). We hypothesize that AgNPs are taken up by stem cells, but due to technical limitations of the confocal technique, we cannot exclude NP signals emerging from above or below the focal plane. Several in vitro studies have assessed the effects of AgNPs on stem cells, with contradictory results. Inhibitory effects on stem cell proliferation were found for non-coated AgNPs (Liu et al., 2015) and PVP-coated AgNPs (Greulich et al., 2009, He et al., 2016a) in various stem cell types. Other studies detected an inhibition of differentiation by non-coated AgNPs (Park et al., 2011, Sengstock et al., 2014). However, several studies reported that exposure to AgNPs did not alter (Samberg et al., 2012, Liu et al., 2014b, He et al., 2016b) or even stimulate (Qin et al., 2014, Ong et al., 2016) stem cell differentiation. Differences can be related to the different set-up, again emphasizing the importance of physicochemical and toxicokinetic characterization in each experiment. Only this way, potential risks can be properly estimated, for example by including these properties as an additional parameter to assess toxicity in vivo, and explain heterogeneity in toxic effects. In any case, the variability in healing and toxicity potential are to be considered in AgNP applications, especially for smaller particle sizes and in case of PVP coating. These can be taken up by stem and early progenitor cells, as suggested by our data.

Another parameter interfering with AgNP toxicity is tissue heterogeneity. Heads were more sensitive to AgNPs, resulting in a stronger mitotic delay, potentially caused by more fragmented DNA (Figure S7(B)). Also head regeneration (i.e. regenerating a new head from a tail part), and especially the formation of the cephalic ganglia, was delayed, with more 
pronounced results after PVP-AgNP exposure (Figure 2(D)). This is of added value to existing literature, as most studies on neurodevelopmental effects of AgNPs were performed in vitro (Liu et al., 2015, Repar et al., 2018, Yamada et al., 2018). Similar to what was previously demonstrated (Pirotte et al., 2015), a well-balanced redox homeostasis is needed to regulate stem cell differentiation rather than proliferation or maintenance in regenerating tails (Figure S6). Organisms with delayed regeneration due to AgNP exposure were 'rescued' by interfering with the redox system. By inhibiting SOD, superoxide degradation and hydrogen peroxide production rates are altered, thereby counteracting a potential disbalance. We further hypothesize that also other antioxidant mechanisms are activated during AgNP exposure, to compensate SOD inhibition (Abdal Dayem et al., 2017) (Figure 4(A)).

Our experiments on homeostatic animals again emphasized AgNP toxicity towards developing tissues (Figure 5). Not even the highest concentrations (up to $50 \mathrm{mg} / \mathrm{l}$ for 7 days) led to effects on stem cell proliferation or brain ganglia in the homeostatic animals. We did observe behavioral effects which might indicate neurodegeneration, but this could not be confirmed at tissue level (Figure 5(B)). It is possible that more subtle effects on specific neuronal populations were missed. We hypothesize that developing organisms are more sensitive to AgNPs, because of the important role of proliferating (stem) cells in early development, which functioning relies on redox-related changes (Liu et al., 2014a). A higher sensitivity of stem cells compared to differentiated cells was reported earlier for zinc oxide NPs (Taccola et al., 2011), a metallic NP with redox balance disturbing potential (Zhao et al., 2016). Recently, Zuberek et al. (2018) showed that proliferating human neuronal cells are far more susceptible to AgNPs (20 nm) compared to differentiated neurons. Silver particlespecific toxicity towards proliferating cells can prove useful in the destruction of cancerous proliferating cells (AshaRani et al., 2009). 
To conclude, the sensitivity of regenerating organisms to AgNPs demonstrated in this study suggests careful handling of AgNP in applications for healing or developing tissues. The AgNP-induced effects on stem cell dynamics and the delayed neuroregeneration indicate that it is essential to include tissue heterogeneity and nanoparticle characteristics in future risk assessment. AgNP characteristics influenced subcellular effects such as the severity of DNA damage and characteristics changed during exposure, forming agglomerates and precipitating out of suspension. Based on these results, we want to emphasize the importance of characterizing NP properties in different settings, and strongly suggest to take these characteristics into account in toxicity studies.

\section{Declaration of interest statement}

The authors declare no competing interests. 


\section{References}

Abdal Dayem, A., Hossain, K. M., Lee, B. S., Kim, K., Saha, K. S., Yang, G.-M., Choi, Y. H. and Cho, S.-G. 2017. The Role of Reactive Oxygen Species (ROS) in the Biological Activities of Metallic Nanoparticles. International Journal of Molecular Sciences, 18 (1), 120.

Abramenko, N. B., Demidova, T. B., Abkhalimov, E. V., Ershov, B. G., Krysanov, E. Y. and Kustov, L. M. 2018. Ecotoxicity of different-shaped silver nanoparticles: Case of zebrafish embryos. Journal of Hazardous Materials, 347, 89-94.

Ahamed, M., Karns, M., Goodson, M., Rowe, J., Hussain, S. M., Schlager, J. J. and Hong, Y. 2008. DNA damage response to different surface chemistry of silver nanoparticles in mammalian cells. Toxicology and Applied Pharmacology, 233 (3), 404-410.

Almeida, J. P., Chen, A. L., Foster, A. and Drezek, R. 2011. In vivo biodistribution of nanoparticles. Nanomedicine, 6 (5), 815-35.

Archana, D., Singh, B. K., Dutta, J. and Dutta, P. K. 2015. Chitosan-PVP-nano silver oxide wound dressing: in vitro and in vivo evaluation. International Journal of Biological Macromolecules, 73, 49-57.

Asharani, P., Hande, M. P. and Valiyaveettil, S. 2009. Anti-proliferative activity of silver nanoparticles. BMC Cell Biology, 10 (1), 65.

Asharani, P. V., Lianwu, Y., Gong, Z. and Valiyaveettil, S. 2011. Comparison of the toxicity of silver, gold and platinum nanoparticles in developing zebrafish embryos. Nanotoxicology, 5 (1), $43-$ 54.

Baccaro, M., Undas, A. K., De Vriendt, J., Van Den Berg, J. H. J., Peters, R. J. B. and Van Den Brink, N. W. 2018. Ageing, dissolution and biogenic formation of nanoparticles: how do these factors affect the uptake kinetics of silver nanoparticles in earthworms? Environmental Science: Nano, 5 (5), 1107-1116.

Baguñà, J. 1976. Mitosis in the intact and regenerating planarian Dugesia mediterranea n.sp. I. Mitotic studies during growth, feeding and starvation. Journal of Experimental Zoology, 195 (1), 53-64.

Bantz, C., Koshkina, O., Lang, T., Galla, H.-J., Kirkpatrick, C. J., Stauber, R. H. and Maskos, M. 2014. The surface properties of nanoparticles determine the agglomeration state and the size of the particles under physiological conditions. Beilstein Journal of Nanotechnology, 5, 1774-1786.

Behra, R., Sigg, L., Clift, M. J. D., Herzog, F., Minghetti, M., Johnston, B., Petri-Fink, A. and RothenRutishauser, B. 2013. Bioavailability of silver nanoparticles and ions: from a chemical and biochemical perspective. Journal of the Royal Society Interface, 10 (87), 20130396.

Benn, T., Cavanagh, B., Hristovski, K., Posner, J. D. and Westerhoff, P. 2010. The release of nanosilver from consumer products used in the home. Journal of Environmental Quality, 39 (6), 187582.

Bergin, I. L. and Witzmann, F. A. 2013. Nanoparticle toxicity by the gastrointestinal route: evidence and knowledge gaps. International journal of biomedical nanoscience and nanotechnology, 3 (1-2), 163-210.

Bilberg, K., Hovgaard, M. B., Besenbacher, F. and Baatrup, E. 2012. In Vivo Toxicity of Silver Nanoparticles and Silver lons in Zebrafish (Danio rerio). Journal of Toxicology, 2012.

Carbone, M., Donia, D. T., Sabbatella, G. and Antiochia, R. 2016. Silver nanoparticles in polymeric matrices for fresh food packaging. Journal of King Saud University - Science.

Chen, X. and Schluesener, H. J. 2008. Nanosilver: A nanoproduct in medical application. Toxicology Letters, $176(1), 1-12$.

Cho, Y.-M., Mizuta, Y., Akagi, J.-I., Toyoda, T., Sone, M. and Ogawa, K. 2018. Size-dependent acute toxicity of silver nanoparticles in mice. Journal of Toxicologic Pathology, 31 (1), 73-80.

Courrier, H. M., Butz, N. and Vandamme, T. F. 2002. Pulmonary drug delivery systems: recent developments and prospects. Critical Reviews in Therapeutic Drug Carrier Systems, 19 (4-5), 425-98. 
Dakal, T. C., Kumar, A., Majumdar, R. S. and Yadav, V. 2016. Mechanistic Basis of Antimicrobial Actions of Silver Nanoparticles. Frontiers in Microbiology, 7, 1831.

De Lima, R., Seabra, A. B. and Duran, N. 2012. Silver nanoparticles: a brief review of cytotoxicity and genotoxicity of chemically and biogenically synthesized nanoparticles. Journal of Applied Toxicology, 32 (11), 867-879.

Eisenhoffer, G. T., Kang, H. and Alvarado, A. S. 2008. Molecular Analysis of Stem Cells and Their Descendants during Cell Turnover and Regeneration in the Planarian Schmidtea mediterranea. Cell Stem Cell, 3 (3), 327-339.

El Yamani, N., Collins, A. R., Runden-Pran, E., Fjellsbo, L. M., Shaposhnikov, S., Zienolddiny, S. and Dusinska, M. 2017. In vitro genotoxicity testing of four reference metal nanomaterials, titanium dioxide, zinc oxide, cerium oxide and silver: towards reliable hazard assessment. Mutagenesis, 32 (1), 117-126.

Elechiguerra, J. L., Burt, J. L., Morones, J. R., Camacho-Bragado, A., Gao, X., Lara, H. H. and Yacaman, M. J. 2005. Interaction of silver nanoparticles with HIV-1. Journal of Nanobiotechnology, 3, 6.

Fabrega, J., Luoma, S. N., Tyler, C. R., Galloway, T. S. and Lead, J. R. 2011. Silver nanoparticles: Behaviour and effects in the aquatic environment. Environment International, 37 (2), 517531.

Falck, A. J., Mooney, S., Kapoor, S. S., White, K. M. R., Bearer, C. and El Metwally, D. 2015. Developmental Exposure to Environmental Toxicants. Pediatric Clinics of North America, 62 (5), 1173-1197.

Gentile, L., Cebrià, F. and Bartscherer, K. 2011. The planarian flatworm: an in vivo model for stem cell biology and nervous system regeneration. Disease Models \& Mechanisms, 4 (1), 12-19.

Goupil, L. S., Ivry, S. L., Hsieh, I., Suzuki, B. M., Craik, C. S., O'donoghue, A. J. and Mckerrow, J. H. 2016. Cysteine and Aspartyl Proteases Contribute to Protein Digestion in the Gut of Freshwater Planaria. PLoS Neglected Tropical Diseases, 10 (8).

Gray, E. P., Coleman, J. G., Bednar, A. J., Kennedy, A. J., Ranville, J. F. and Higgins, C. P. 2013. Extraction and Analysis of Silver and Gold Nanoparticles from Biological Tissues Using Single Particle Inductively Coupled Plasma Mass Spectrometry. Environmental Science \& Technology, 47 (24), 14315-14323.

Greulich, C., Kittler, S., Epple, M., Muhr, G. and Koller, M. 2009. Studies on the biocompatibility and the interaction of silver nanoparticles with human mesenchymal stem cells (hMSCs). Langenbecks Archives of Surgery, 394 (3), 495-502.

Guo, D., Zhu, L., Huang, Z., Zhou, H., Ge, Y., Ma, W., Wu, J., Zhang, X., Zhou, X., Zhang, Y., Zhao, Y. and $\mathrm{Gu}$, N. 2013. Anti-leukemia activity of PVP-coated silver nanoparticles via generation of reactive oxygen species and release of silver ions. Biomaterials, 34 (32), 7884-7894.

Hagstrom, D., Cochet-Escartin, O. and Collins, E. M. 2016. Planarian brain regeneration as a model system for developmental neurotoxicology. Regeneration, 3 (2), 65-77.

Hagstrom, D., Cochet-Escartin, O., Zhang, S., Khuu, C. and Collins, E. S. 2015. Freshwater planarians as an alternative animal model for neurotoxicology. Toxicological Sciences.

He, W., Kienzle, A., Liu, X., Müller, W. E. G., Elkhooly, T. A. and Feng, Q. 2016a. In Vitro Effect of 30 $\mathrm{nm}$ Silver Nanoparticles on Adipogenic Differentiation of Human Mesenchymal Stem Cells. Journal of Biomedical Nanotechnology, 12 (3), 525-535.

He, W., Liu, X., Kienzle, A., Müller, W. E. G. and Feng, Q. 2016b. In Vitro Uptake of Silver Nanoparticles and Their Toxicity in Human Mesenchymal Stem Cells Derived from Bone Marrow. Journal of Nanoscience and Nanotechnology, 16 (1), 219-228.

Inden, M., Kitamura, Y., Taniguchi, T., Watanabe, K. and Agata, K. 2004. Parkinsonian model of planarian, an invertebrate flatworm. International Congress Series, 1260, 291-295.

King, R. and Newmark, P. 2013. In situ hybridization protocol for enhanced detection of gene expression in the planarian Schmidtea mediterranea. BMC Developmental Biology, 13 (1), 8. 
Kumar, S. S. D., Rajendran, N. K., Houreld, N. N. and Abrahamse, H. 2018. Recent advances on silver nanoparticle and biopolymer based biomaterials for wound healing applications. International Journal of Biological Macromolecules.

Kustov, L., Tiras, K., Al-Abed, S., Golovina, N. and Ananyan, M. 2014. Estimation of the toxicity of silver nanoparticles by using planarian flatworms. Alternatives to Lab Animals, 42 (1), 51-58.

Lee, H. Y., Park, H. K., Lee, Y. M., Kim, K. and Park, S. B. 2007. A practical procedure for producing silver nanocoated fabric and its antibacterial evaluation for biomedical applications. Chemical Communications, (28), 2959-2961.

Li, X., Lenhart, J. J. and Walker, H. W. 2010. Dissolution-Accompanied Aggregation Kinetics of Silver Nanoparticles. Langmuir, 26 (22), 16690-16698.

Liu, F., Mahmood, M., Xu, Y., Watanabe, F., Biris, A. S., Hansen, D. K., Inselman, A., Casciano, D., Patterson, T. A., Paule, M. G., Slikker, W., Jr. and Wang, C. 2015. Effects of silver nanoparticles on human and rat embryonic neural stem cells. Frontiers in Neuroscience, 9.

Liu, J. C., Lerou, P. H. and Lahav, G. 2014a. Stem cells: balancing resistance and sensitivity to DNA damage. Trends in Cell Biology, 24 (5), 268-274.

Liu, X., He, W., Fang, Z., Kienzle, A. and Feng, Q. 2014b. Influence of silver nanoparticles on osteogenic differentiation of human mesenchymal stem cells. Journal of Biomedical Nanotechnology, 10 (7), 1277-1285.

Liu, X., Xu, Y., Wu, Z. and Chen, H. 2013. Poly(N-vinylpyrrolidone)-modified surfaces for biomedical applications. Macromolecular Bioscience, 13 (2), 147-154.

Mcgillicuddy, E., Murray, I., Kavanagh, S., Morrison, L., Fogarty, A., Cormican, M., Dockery, P., Prendergast, M., Rowan, N. and Morris, D. 2017. Silver nanoparticles in the environment: Sources, detection and ecotoxicology. Science of The Total Environment, 575, 231-246.

Mcshan, D., Ray, P. C. and Yu, H. 2014. Molecular toxicity mechanism of nanosilver. Journal of Food and Drug Analysis, 22 (1), 116-127.

Mehennaoui, K., Georgantzopoulou, A., Felten, V., Andreï, J., Garaud, M., Cambier, S., Serchi, T., PainDevin, S., Guérold, F., Audinot, J.-N., Giambérini, L. and Gutleb, A. C. 2016. Gammarus fossarum (Crustacea, Amphipoda) as a model organism to study the effects of silver nanoparticles. Science of The Total Environment, 566-567, 1649-1659.

Neal-Kluever, A., Aungst, J., Gu, Y., Hatwell, K., Muldoon-Jacobs, K., Liem, A., Ogungbesan, A. and Shackelford, M. 2014. Infant toxicology: State of the science and considerations in evaluation of safety. Food and Chemical Toxicology, 70, 68-83.

Nguyen, K., L Seligy, V., Massarsky, A., Moon, T., Rippstein, P., Jtan and F Tayabali, A. 2013. Comparison of toxicity of uncoated and coated silver nanoparticles.

Ong, C., Lee, Q. Y., Cai, Y., Liu, X., Ding, J., Yung, L.-Y. L., Bay, B.-H. and Baeg, G.-H. 2016. Silver nanoparticles disrupt germline stem cell maintenance in the Drosophila testis. Scientific Reports, 6 (20632).

Pakrashi, S., Tan, C. and Wang, W. X. 2017. Bioaccumulation-based Silver Nanoparticle Toxicity in Daphnia Magna and Maternal Impacts. Environmental toxicology and chemistry, 36 (12), 3359-3366.

Park, M. V. D. Z., Neigh, A. M., Vermeulen, J. P., De La Fonteyne, L. J. J., Verharen, H. W., Briedé, J. J., Van Loveren, H. and De Jong, W. H. 2011. The effect of particle size on the cytotoxicity, inflammation, developmental toxicity and genotoxicity of silver nanoparticles. Biomaterials, 32 (36), 9810-9817.

Perera, F. and Herbstman, J. 2011. Prenatal environmental exposures, epigenetics, and disease. Reproductive Toxicology, 31 (3), 363-373.

Peters, R. J. B., Van Bemmel, G., Milani, N. B. L., Den Hertog, G. C. T., Undas, A. K., Van Der Lee, M. and Bouwmeester, H. 2018. Detection of nanoparticles in Dutch surface waters. Science of the Total Environment, 621, 210-218.

Pirotte, N., Stevens, A. S., Fraguas, S., Plusquin, M., Van Roten, A., Van Belleghem, F., Paesen, R., Ameloot, M., Cebria, F., Artois, T. and Smeets, K. 2015. Reactive Oxygen Species in Planarian 
Regeneration: An Upstream Necessity for Correct Patterning and Brain Formation. Oxid Med Cell Longev, 2015, 392476.

Qin, H., Zhu, C., An, Z., Jiang, Y., Zhao, Y., Wang, J., Liu, X., Hui, B., Zhang, X. and Wang, Y. 2014. Silver nanoparticles promote osteogenic differentiation of human urine-derived stem cells at noncytotoxic concentrations. International journal of nanomedicine, 9, 2469-2478.

Reddien, P. W., Oviedo, N. J., Jennings, J. R., Jenkin, J. C. and Alvarado, A. S. 2005. SMEDWI-2 Is a PIWI-Like Protein That Regulates Planarian Stem Cells. Science, 310 (5752), 1327.

Repar, N., Li, H., Aguilar, J. S., Li, Q. Q., Drobne, D. and Hong, Y. 2018. Silver nanoparticles induce neurotoxicity in a human embryonic stem cell-derived neuron and astrocyte network. Nanotoxicology, 1-13.

Romih, T., Jemec, A., Kos, M., Hočevar, S. B., Kralj, S., Makovec, D. and Drobne, D. 2016. The role of PVP in the bioavailability of Ag from the PVP-stabilized Ag nanoparticle suspension. Environmental Pollution, 218, 957-964.

Salvetti, A., Rossi, L., lacopetti, P., Li, X., Nitti, S., Pellegrino, T., Mattoli, V., Golberg, D. and Ciofani, G. 2015. In vivo biocompatibility of boron nitride nanotubes: effects on stem cell biology and tissue regeneration in planarians. Nanomedicine, 10 (12), 1911-1922.

Samberg, M. E., Loboa, E. G., Oldenburg, S. J. and Monteiro-Riviere, N. A. 2012. Silver nanoparticles do not influence stem cell differentiation but cause minimal toxicity. Nanomedicine, 7 (8), 1197-1209.

Scown, T. M., Santos, E. M., Johnston, B. D., Gaiser, B., Baalousha, M., Mitov, S., Lead, J. R., Stone, V., Fernandes, T. F., Jepson, M., Van Aerle, R. and Tyler, C. R. 2010. Effects of aqueous exposure to silver nanoparticles of different sizes in rainbow trout. Toxicological Sciences, 115 (2), 521 534.

Sengstock, C., Diendorf, J., Epple, M., Schildhauer, T. A. and Koller, M. 2014. Effect of silver nanoparticles on human mesenchymal stem cell differentiation. Beilstein Journal of Nanotechnology, 5, 2058-2069.

Stevens, A.-S., Wouters, A., Ploem, J.-P., Pirotte, N., Van Roten, A., Willems, M., Hellings, N., Franken, C., Koppen, G., Artois, T., Plusquin, M. and Smeets, K. 2017. Planarians Customize Their Stem Cell Responses Following Genotoxic Stress as a Function of Exposure Time and Regenerative State. Toxicological Sciences.

Taccola, L., Raffa, V., Riggio, C., Vittorio, O., Iorio, M. C., Vanacore, R., Pietrabissa, A. and Cuschieri, A. 2011. Zinc oxide nanoparticles as selective killers of proliferating cells. International Journal of Nanomedicine, 6, 1129-1140.

Van Der Zande, M., Vandebriel, R. J., Van Doren, E., Kramer, E., Herrera Rivera, Z., Serrano-Rojero, C. S., Gremmer, E. R., Mast, J., Peters, R. J. B., Hollman, P. C. H., Hendriksen, P. J. M., Marvin, H. J. P., Peijnenburg, A. a. C. M. and Bouwmeester, H. 2012. Distribution, Elimination, and Toxicity of Silver Nanoparticles and Silver lons in Rats after 28-Day Oral Exposure. American Chemical Society, Nano, 6 (8), 7427-7442.

Yamada, S., Yamazaki, D. and Kanda, Y. 2018. Silver nanoparticles inhibit neural induction in human induced pluripotent stem cells. Nanotoxicology, 1-11.

Zhao, C. M. and Wang, W. X. 2010. Biokinetic uptake and efflux of silver nanoparticles in Daphnia magna. Environmental Science and Technology, 44 (19), 7699-7704.

Zhao, C. M. and Wang, W. X. 2011. Comparison of acute and chronic toxicity of silver nanoparticles and silver nitrate to Daphnia magna. Environmental toxicology and chemistry, 30 (4), 885892.

Zhao, X., Ren, X., Zhu, R., Luo, Z. and Ren, B. 2016. Zinc oxide nanoparticles induce oxidative DNA damage and ROS-triggered mitochondria-mediated apoptosis in zebrafish embryos. Aquatic Toxicology, 180, 56-70.

Zhu, M., Nie, G., Meng, H., Xia, T., Nel, A. and Zhao, Y. 2013. Physicochemical properties determine nanomaterial cellular uptake, transport and fate. Accounts of chemical research, 46 (3), 622631. 
Zuberek, M., Stepkowski, T. M., Kruszewski, M. and Grzelak, A. 2018. Exposure of human neurons to silver nanoparticles induces similar pattern of $A B C$ transporters gene expression as differentiation: Study on proliferating and post-mitotic LUHMES cells. Mech Ageing Dev. 